\title{
émulations
}

\section{Le pétrole, entre enjeux économiques et enjeux politiques}

\author{
François Pelletier
}

Émulations - Revue de sciences sociales

$2018, n^{\circ} 26$, « Repenser la dichotomie 'national vs international' »

\section{Article disponible à l'adresse suivante}

https://ojs.uclouvain.be/index.php/emulations/article/view/pelletier

\section{Pour citer cet article}

Pelletier François, "Le pétrole, entre enjeux économiques et enjeux politiques ». Émulations, $N^{\circ} 26$, pp. 51-62. Mise en ligne le 15 décembre 2018.

DOI : 10.14428/emulations.026.03

Distribution électronique : Université catholique de Louvain (Belgique) : ojs.uclouvain.be

(C) Cet article est mis à disposition selon les termes de la Licence Creative Commons Attribution, Pas d'Utilisation Commerciale 4.0 International. http://creativecommons.org/licenses/by-nc/4.0/

Éditeur : Émulations - Revue de sciences sociales / Presses universitaires de Louvain https://ojs.uclouvain.be/index.php/emulations

ISSN électronique : 1784-5734

UCL PRESSES

UNIVERSITAIRES

DE LOUVAIN 


\title{
Le pétrole, entre enjeux économiques et enjeux politiques
}

François Pelletier ${ }^{1}$

\begin{abstract}
[Résumé] Cet article retrace une évolution économique majeure au lendemain de la Première Guerre mondiale. L'utilisation croissante du pétrole a de nombreuses répercussions sur les sociétés industrielles qui en dépendent de plus en plus pour leur approvisionnement. Son commerce engendre de nouvelles tensions entre espaces nationaux et dynamiques d'internationalisation. L'article expose les mécanismes de ce changement et l'élaboration de politiques nationales et internationales en France. II permet de cerner l'impact stratégique du pétrole, mais aussi son rôle dans le cadre d'une transformation libérale de l'économie mondiale. Sur la scène internationale, il n'est pas exagéré de dire que la hantise du pétrole occupe le cauchemar des chancelleries. Les États-nations entrent désormais en lice afin d'assurer leur approvisionnement. À l'échelle nationale, cette interaction se fait aux dépens du libéralisme et conduit à l'adoption de politiques économiques et industrielles. Cette évolution recoupe des enjeux de politique internationale et de sécurité, des enjeux industriels, technologiques et économiques qui impliquent des acteurs publics et privés, nationaux et internationaux.
\end{abstract}

Mots-clés : pétrole ; relations internationales ; France ; histoire économique ; entredeux-guerres.

[Abstract] This article analyses a major economic development which occurred at the end of the First World War. The use of oil as a commodity had many repercussions on industrial countries which were increasingly dependent on it for their supply. The trade of this precious fuel engendered new tensions between national spaces and fostered new international dynamics. This article exposes the mechanism of this change and the formulation of national and international policies in France. It allows us to grasp the strategic impact of oil, but also its role in a liberal transformation of the world economy. On the international scene, oil had diplomats worried. Governments now competed for access to supplies. On the national stage, liberal values are sidelined in favor of economic regulations. These are issues of international politics and security, of technology, industrial and economic knowhow. They include private and public actors, on a national and an international level.

Keywords: oil; international relations; France; economic history; inter-war.

L'exploitation du pétrole engendre de nouveaux modes de consommation dès la fin du XIXe siècle. Son utilisation, d'abord pour l'éclairage, s'impose rapidement à travers le monde. Au début du siècle suivant, on lui découvre un potentiel nouveau. Son pouvoir calorifique double le rayon d'action des navires et sa fluidité diminue le temps de ravitaillement (Vaisset, 2012 : 73). L'automobile lui assure un marché en pleine croissance.

\footnotetext{
${ }^{1}$ Université de Montréal et Université Paris 1 Panthéon-Sorbonne.
} 
Ces considérations engendrent de nouveaux besoins dont les répercussions sont importantes pour les économies nationales des pays consommateurs. La Première Guerre mondiale fait émerger au premier plan le caractère stratégique de cette matière première, dont dépend désormais la sécurité nationale. L'État intervient désormais au nom de l'intérêt national. Son intervention est protéiforme ; il dispose de trois moyens d'intervention : la loi, l'argent et la diplomatie. La loi lui permet de poser les règles du jeu. L'intervention financière est une arme puissante, mais mal perçue par les milieux d'affaires. Finalement, l'action diplomatique est nécessaire pour le partage mondial des ressources et pour l'accaparement des zones de prospection les plus favorables (Bouguen, 2013: 27).

Au début du XXe siècle, ces outils sont utilisés de manières très différentes par les puissances industrielles. Les gouvernements français, anglais et américains développent des politiques nationales distinctes. Cet article retrace la position française et la stratégie adoptée par l'Hexagone au début du siècle. La France représente alors un marché lucratif pour les compagnies pétrolières. Nation industrielle avancée, elle nécessite des quantités de pétrole sans cesse croissantes pour combler ses besoins en énergie. Les responsables politiques français sont au courant du danger que leur fait courir cette position. Les importations de pétrole pèsent lourd sur la balance commerciale. L'action du gouvernement est à replacer dans ce contexte de crise. Afin d'analyser cette conjoncture, l'article s'appuie sur une palette d'archives françaises, publiques et privées. La confrontation de ces documents est révélatrice lorsqu'il s'agit d'évaluer la formulation et l'implantation de politiques pétrolières en France. L'interaction entre États-nations et international, engendrée par l'utilisation croissante du pétrole, est passée au peigne fin. L'importance prise par les compagnies pétrolières et les caractéristiques inhérentes à ce secteur signifient que le cadre de leur intervention dépasse l'échelle nationale. Ces sociétés sont des acteurs à part entière et demeurent étroitement mêlées à la vie politique, où elles font figure de puissances. Ces géants du pétrole accumulent un capital imposant et leur influence se fait aussi sentir dans les corridors des chancelleries. Ce sont les premières multinationales et les précurseurs de la mondialisation. La politique pétrolière des États-nations ne se fait donc pas en vase clos et la France n'est pas une exception. Elle doit composer avec ces géants industriels dont l'action transcende les frontières. Cette interaction se fait rarement sans heurt et les gouvernements résistent déjà aux logiques mondialisatrices dont les prémices symbolisent une perte d'influence certaine. Cet article retrace donc une évolution importante dans le rapport entre le national et l'international au début du XX' siècle.

\section{Le pétrole dans les relations internationales}

La structure de l'économie mondiale connaît de profondes mutations au lendemain de la Première Guerre mondiale. La guerre altère l'importance du pétrole, celui-ci est désormais au cœur des débats diplomatiques. Globalement, la fin du conflit marque le 
déclin de l'Empire britannique et l'ascendance américaine, mais lorsqu'il s'agit de pétrole les répercussions de ce changement ne se font pas sentir dans l'immédiat. Elles accusent même un certain retard par rapport à l'évolution économique générale (BullBerg, 1987:141). L'ordre international assure à l'Angleterre la mainmise sur les plus gros gisements pétroliers. Le contrôle des territoires, étendu après la guerre grâce au système des mandats, ferme d'importantes sections du globe aux investissements étrangers. Cet ordre mondial ne convient pas aux intérêts américains. Les États-Unis visent à briser la dominance britannique et à ouvrir le monde à la concurrence. En matière de politique étrangère, le pétrole se conforme bien aux principes américains dont l'objectif est l'égalité d'accès et de traitement pour ses nationaux à travers le monde. Cette politique est celle de la " Porte ouverte », au nom de laquelle les États-Unis prônent la mise en place d'un ordre économique libéral. Cette poussée s'inscrit dans les prémices de la mondialisation. En termes d'accès aux ressources pétrolières, elle vise des changements majeurs. C'est la question diplomatique la plus importante dans la décennie qui suit la fin de la guerre.

Aux États-Unis, les années 1920 sont marquées par la crainte d'une importante pénurie de pétrole. Cette appréhension pousse les compagnies pétrolières américaines à acquérir de nouveaux gisements outre-mer. Cette percée ne se heurte pas uniquement aux intérêts britanniques, mais aussi au nationalisme émergent dans les pays producteurs et certains pays consommateurs comme la France. Aux États-Unis, cette expansion se fait dans le cadre d'un partenariat entre le gouvernement et l'industrie. L'expansionnisme économique correspond aux intérêts de l'un et l'autre. Pour le premier, l'accès aux ressources outre-mer est une question de sécurité nationale. Pour le second, c'est une question de marché et de profits. Ce modèle de coopération est durable aux États-Unis et tend à maximiser les investissements étrangers effectués par le secteur privé. Il s'oppose aux modèles interventionnistes qui seront appliqués en France ou en Angleterre. Washington tend plutôt à assurer la compétitivité de son industrie à travers le monde, dans le cadre d'un système de libre-échange. Dès la fin de l'administration Wilson, la politique pétrolière américaine est fermement établie selon ce principe de coopération avec l'industrie.

En matière de pétrole, la rivalité entre l'Angleterre et les États-Unis domine l'entredeux-guerres. Au sens large, l'enjeu est alors de redéfinir la structure de l'économie mondiale et d'ouvrir les frontières. En Angleterre, la plupart des responsables politiques étaient réticents à lever les restrictions coloniales. Vu l'absence de ressources pétrolières sur le territoire national, l'accès préférentiel à des gisements dans les colonies revêtait une importance particulière pour le gouvernement britannique. Un conflit d'intérêts avec l'Amérique semblait donc inévitable. Il apparaissait difficile de concilier l'intérêt d'une nation responsable de $70 \%$ de la production mondiale à partir de son propre territoire (États-Unis) avec celui d'une nation produisant seulement $3 \%$ de ses besoins, tout en étant un des plus importants consommateurs (Royaume-Uni). Ni le 
gouvernement britannique ni les compagnies n'étaient prêts à accepter une participation américaine dans le golfe persique. Dans les Indes orientales, l'Angleterre est plus sympathique envers la position américaine. Elle favorise une coopération afin d'écarter la compétition allemande ou japonaise. C'est donc une position essentiellement pragmatique, même si l'Angleterre maintient son opposition au renversement de l'ordre établi. L'ouverture des frontières aux intérêts américains se fait pour des raisons stratégiques ou pour éviter une compétition excessive et une perturbation des prix. Il est tout de même remarquable qu'en pleine période isolationniste, les États-Unis aient agi, avec une conception claire de leur intérêt national, afin de défendre leurs nationaux outre-mer. Ces efforts entraînent le pays davantage « dans le vortex d'une expansion mondiale » (Randall, 2005 : 29).

C'est dans ce contexte global que naît la politique pétrolière française, alors que les relations anglo-américaines sont l'axe autour duquel tourne toute la diplomatie en matière de pétrole. La France est au centre d'un marché en pleine expansion, sa consommation de produits pétroliers croît sans interruption durant l'entre-deux-guerres. Dans l'Hexagone, la guerre a un effet-choc sur l'action politique. Entièrement dépendante de ses alliés, autant pour la fourniture que pour le transport des produits pétroliers, le pays frôle la catastrophe en décembre 1917 alors que son approvisionnement ne parvient plus à couvrir ses besoins (Nayberg, 1997 : 77). La crise pousse l'État à intervenir. Un Comité général du pétrole est créé le 13 juillet 1917 pour diriger les opérations pétrolières. L'épuisement des stocks aboutit ensuite à la naissance du Consortium français d'importation de pétrole et d'essence. Sous ce régime, l'État détient le monopole des importations. Les motivations qui poussent la France à agir dans le secteur pétrolier sont claires au lendemain de la guerre. Elle réalise être entièrement dépendante de ses alliés autant pour la fourniture que pour le transport des produits pétroliers. À l'international, cette volonté se heurte à une dure réalité. La France se présente bien tard sur un marché étroitement contrôlé par de puissants trusts. Les choix sont donc limités pour la diplomatie française. Le principe américain de la Porte ouverte ne lui convient pas, puisqu'aucune société française n'a les moyens de mener une politique indépendante. La France peut, comme l'Angleterre, miser sur ses possessions coloniales et y chercher du pétrole. C'est une entreprise risquée et coûteuse. C'est pourtant le pari que prennent les responsables français au lendemain de la guerre. Durant l'entre-deuxguerres, l'exploration s'étend au Maroc, en Tunisie, en Algérie et au Gabon. Malgré des millions de francs dépensés, les recherches menées dans l'empire français s'avèrent être un échec cuisant.

Dans l'immédiat de l'après-guerre, le manque de ressources financières et le potentiel que représentent les colonies sont un obstacle à un rapprochement franco-américain. La précarité du Trésor public, sous-pression pour la reconstruction des régions dévastées, contribue au fait que la France n'a pas les moyens de ses ambitions (Kuisel, 1967: 30). Faute de pouvoir concurrencer les grands trusts du pétrole sur la scène internationale, Paris défend l'ordre établi. Ce choix apparaît au grand jour lors de l'accord de San 
Remo. L'entente, signée le 24 avril 1920, est le fondement de la politique pétrolière française après la guerre. La France se positionne clairement du côté de l'Angleterre. Le traité prévoit une coopération des deux puissances en Roumanie, en Asie Mineure, dans les territoires de l'ancien Empire russe, en Galicie et dans les colonies françaises et britanniques. C'est une véritable alliance pétrolière, elle garantit aussi à la France une part de $25 \%$ dans les pétroles de Mésopotamie. Cet accord vient renforcer l'ordre international établi, au grand dam des Américains dont les protestations sont immédiates. Elle confirme la politique britannique qui est de s'entendre directement avec l'État français pour établir une relation privilégiée.

L'accord de San Remo aboutit à des résultats concrets pour la France. En Mésopotamie, la participation obtenue dans l'Iraq Petroleum Company (IPC) assure à l'Hexagone une part dans les grandes affaires de pétrole à l'international. Ce sont des droits importants sur les affaires pétrolières au Moyen-Orient, alors que même les États-Unis sont toujours exclus de la région. Pendant les huit années qui suivent la conférence de San Remo, les compagnies américaines s'appuient sur le principe de la Porte ouverte pour entrer au Moyen-Orient. Elles mènent des négociations avec deux compagnies anglaises, l'Anglo-Persian et Royal-Dutch, afin d'être admises au sein de l'IPC. Le Foreign Office n'est pas insensible à ces revendications de la part d'un allié dont la participation s'est avérée décisive durant la Première Guerre mondiale. Les Américains s'attendent à ce qu'une entente comprenne le même traitement pour chacun des membres. L'Angleterre voit plutôt la cession de parts comme une faveur et entend tirer des compensations en échange. C'est dans ces circonstances que la France rejoint la table de négociation en 1924, après la création d'une compagnie nationale chargée de représenter le pays (la Compagnie française des pétroles). Si la politique pétrolière française s'est largement appuyée sur son allié britannique dans ses débuts, les premières négociations avec les Américains signalent un important réalignement. Il existe alors d'importants intérêts français œuvrant en faveur d'une alliance franco-américaine. Dans les milieux d'affaires, la banque de Paris et des Pays-Bas (Paribas) est la plus importante à agir dans ce sens sous l'impulsion de son directeur-général, Horace Finaly. Ce rapprochement doit permettre aux deux groupes d'agir de concert pour réduire les prétentions anglaises à des proportions raisonnables et permettre l'entrée des Américains dans la compagnie. Ce raisonnement s'appuie sur le fait que les deux compagnies anglaises membres de l'IPC sont en fait les instruments du gouvernement britannique. C'est, du moins, la rhétorique employée pour justifier un rapprochement. « L’Anglo-Persian, société anglaise avec participation du gouvernement, et la Royal-Dutch [...] sont influencées dans leur conception de la conduite de la [compagnie] par des éléments d'ordre politique auxquels les groupes français et américain sont absolument étrangers².»

En matière de politique pétrolière internationale, le réalignement de la France est surprenant et surtout dangereux. Il se base sur l'idée que les deux compagnies anglaises

\footnotetext{
${ }^{2}$ Archives de la banque Paribas, 3CABET/1/283, Turkish petroleum, 18 juillet 1925, p. 2.
} 
membres de l'IPC forment un bloc soudé, ce qui ne correspond pas à la réalité. Cette analyse est pourtant courante et elle conditionne l'action du groupe français. Le danger d'une telle manœuvre vient du fait qu'une entente avec les Américains risque de passer par une acceptation du principe de la Porte ouverte, c'est-à-dire ouvrir le Moyen-Orient à la concurrence internationale et renoncer aux accords diplomatiques. Or, la France n'a toujours pas les moyens de concurrencer les puissants trusts pétroliers, qui plus est dans un territoire sous juridiction britannique. Le groupe français essuie plusieurs échecs lorsqu'il s'agit de faire entrer les Américains dans l'IPC. En 1925, la situation est telle que le groupe américain renonce tout simplement à entrer dans la compagnie. Le groupe français continue pourtant à tenter de résoudre ce problème ${ }^{3}$. Il aura fallu des années, de nombreux efforts, la menace d'un procès et plusieurs recours diplomatiques pour que les groupes arrivent finalement à une entente. L'accord dit « de la ligne rouge », signé le 31 juillet 1928, définit de façon très précise les liens contractuels des actionnaires de l'IPC. C'est à cette occasion que les Américains se joignent à la compagnie. Les États-Unis gagnent accès au pétrole du Moyen-Orient, mais c'est aussi une victoire pour l'Angleterre puisque le gouvernement américain renonce au principe de la Porte ouverte. L'accord montre que la diplomatie américaine agit déjà par pragmatisme. C'est aussi une reconnaissance des réalités auxquels font face les compagnies pétrolières américaines.

L'accord de San Remo a permis à la France de rejoindre un monde relativement fermé. À la base entièrement dépourvue en pétrole, la France prend désormais part aux grandes négociations pétrolières. La tournure des évènements montre que le gouvernement français s'intéresse de près au développement de cette industrie. La participation française en Mésopotamie est la base de cette politique, mais Paris prend d'autres initiatives durant la période. Au lendemain de la guerre, le gouvernement français évalue la possibilité de se ravitailler en Roumanie. C'est le plus gros producteur de pétrole européen et sa position géographique en fait un enjeu stratégique. L'objectif principal est de couper l'Allemagne de tout approvisionnement en pétrole roumain et d'intégrer "la Roumanie dans un bloc d'alliances de revers centre-européennes, offensives contre l'Allemagne et défensives contre la Russie bolchevique » (Sandu, 1999: 7). En Roumanie, la France peut compter sur l'engouement des banques françaises à se substituer aux intérêts allemands dans les affaires de pétrole. Le Quai d'Orsay appuie ces initiatives et planifie déjà grouper les producteurs et raffineurs français en Roumanie pour que leur action soit « entièrement conforme ${ }^{4}$ » aux vues et aux besoins de la politique française. Cette volonté se heurte aux intérêts des banques françaises qui entendent maintenir

\footnotetext{
${ }^{3}$ Français et Américains communiquent régulièrement à ce sujet. Ibid., Lettre de Mercier à Teagle, 31 décembre 1925.

${ }^{4}$ Archives du ministère des Affaires étrangères et du Développement international (MAE), Direction des affaires politiques et commerciales, Roumanie, 110CPCOM/104.
} 
leur autonomie. En Roumanie, le placement des capitaux français dans le secteur pétrolier est un succès, mais la gestion de ce secteur échappe aux responsables français. Les banques, Paribas en tête, parviennent à déjouer les plans centralisateurs du gouvernement. Si leur présence assure une nouvelle source de ravitaillement, elle ne permet pas à la France de mener sa propre politique pétrolière ${ }^{5}$.

À l'échelle internationale, le succès de l'Hexagone est donc mitigé. La guerre lui a offert d'importantes opportunités dont il a su tirer parti. Les banques françaises sont le fer de lance de cette politique, dont les investissements représentent les plus grosses immobilisations industrielles de l'entre-deux-guerres (Marguerat, Jilek, 1987 : 4). La diplomatie française joue un rôle incontournable lorsqu'il s'agit d'appuyer l'action de ses nationaux à l'étranger. Le résultat est loin d'être négligeable, il garantit une participation française dans tous les pays de l'ancien Empire ottoman. La France s'appuie alternativement sur ses alliés britanniques et américains pour l'acquisition, mais aussi pour la mise en valeur de ses avoirs. Avant l'accord d'Achnacarry en 1928, entente internationale entre les grands trusts du pétrole, la position française évolue dans ce contexte de rivalité anglo-américaine, alors que les États-Unis cherchent à remettre en cause l'ordre international. Cette évolution cache une autre réalité, la précarité du Trésor signifie que Paris doit s'appuyer sur l'action de ses nationaux à l'étranger. La mise en place d'une politique pétrolière nationale s'impose. Il s'agit de protéger le marché français et de donner à la diplomatie française les moyens de son action. La nature du système international rend cette implantation nécessaire. L'État semble être le seul acteur en mesure de fédérer les forces économiques de la nation.

\section{La création d'une politique pétrolière nationale}

La nature des opérations pétrolières laisse donc peu d'options à la France au début du $\mathrm{XX}^{\mathrm{e}}$ siècle. Les accords diplomatiques de l'après-guerre lui ont permis de rejoindre un monde relativement fermé, mais le milieu des affaires n'est pas prêt, dans l'immédiat, à assumer cette tâche. Le secteur de l'énergie révèle l'incapacité des sociétés françaises à concurrencer certaines compagnies monolithiques étrangères. À l'échelle nationale, un effort de rationalisation apparaît nécessaire pour faire face à la compétition mondiale. En France, cette modernisation passe par une concentration industrielle. Les pétroliers français n'ont pas le capital ni l'expertise pour concurrencer efficacement les grands trusts du pétrole. Le processus qui aboutit à la mise en place d'une politique pétrolière en France est donc le fruit d'une lente et difficile gestation. Le gouvernement vise à assurer la sécurité de la nation et l'indépendance énergétique. Pourtant, le résultat dépend de la convergence de ses objectifs avec ceux des compagnies pétrolières. Il

\footnotetext{
${ }^{5}$ L'échec du gouvernement français de fédérer l'action de ses nationaux en Roumanie s'explique notamment par les alliances contractées entre les banques françaises et les compagnies pétrolières internationales (Pelletier 2016, 2017).
} 
serait naïf de croire que la politique mise en place durant l'entre-deux-guerres est un résultat exclusif et rationnel calculé strictement selon l'intérêt national.

Cette volonté, d'agir dans un secteur désormais considéré comme essentiel, ne se concrétise pourtant pas immédiatement au lendemain de la guerre. De 1918 à 1923, des organismes civils tentent de définir ce que doit être la politique pétrolière. De nombreux rapports se succèdent sur la question alors que les élus repoussent onze projets les uns à la suite des autres. Plusieurs options s'offrent à l'État : « le retour à la liberté de marché, le monopole absolu, le monopole partiel, ou la régie intéressée » (Bouguen, 2013 : 120). Ce débat est étroitement lié à la création d'une société nationale et fait la une de la presse. Pour les uns, l'État n'est pas en mesure de mobiliser les compétences et le capital nécessaire afin de développer une politique efficace. Pour les autres, une participation étatique présente un gage de sécurité dans un secteur onéreux en termes d'investissement. Â l'Hémicycle les deux camps s'entendent sur le besoin d'élaborer une politique nationale du pétrole et sur le fait que le marché doit être protégé, mais pas sur la forme que doit prendre cette intervention. Une option en particulier séduit : la création d'une société d'exploitation mixte. En Roumanie, le gouvernement fait une première expérience de la difficulté de regrouper et de canaliser l'action des industriels français. La politique nationale qu'il cherche à mettre en place dépend, une fois de plus, de la conjoncture internationale. Les intérêts anglais et américains sont étroitement liés à ce débat. La politique de l'Angleterre est de s'entendre directement avec l'État français pour établir une relation privilégiée. Elle favorise la création de sociétés franco-britanniques et s'oppose à la liberté de marché qui bénéficie surtout aux Américains. La maîtrise qu'exerce la Standard Oil sur le marché français lui accorde un avantage décisif dans cette lutte pour le contrôle du marché français.

En 1923, le dossier avance sous l'impulsion du président français Raymond Poincaré. Celui-ci opte pour la création d'une compagnie nationale et désigne Ernest Mercier pour la diriger ${ }^{6}$. Ce choix a l'allure d'un compromis. «Il n'y a ni gagnant, ni perdant. Les partisans d'un monopole d'État se félicitent de la création d'un outil permettant de mener une politique autonome. Les défenseurs du libre marché voient une structure affaiblie par un lobbying efficace avant même d'être créée, et contrôlée en partie par des intérêts qui lui sont défavorables » (Bouguen, 2013 : 19). La solution préconisée par Poincaré est celle d'une société à caractère national. Le président entend regrouper les capitaux français au sein d'une compagnie privée qui sera chargée de reprendre les droits acquis à San Remo. Or, la polémique autour de ce projet continue et l'action déstabilisatrice de certains intérêts étrangers se poursuit en France. Walter Teagle, patron de la

\footnotetext{
${ }^{6}$ Le choix de Mercier peut paraître suspect puisqu'il dirige déjà les intérêts pétroliers de Paribas en Roumanie. En fait, celui-ci est connu des responsables français. Il a travaillé pendant la guerre au ministère de l'Armement. On lui reconnaît aussi des grandes capacités d'organisateur, non seulement pour son travail en Roumanie, mais pour le rôle fédérateur qu'il a joué dans le secteur électrique.
} 
Standard Oil, est inquiet et demande à connaître de manière précise les moyens d'intervention de la compagnie dans le commerce du pétrole ${ }^{7}$. Les Américains craignent un monopole ou la mise en place de privilèges particuliers qui pourraient nuire aux sociétés pétrolières existantes dans la continuation de leurs activités. La pression des milieux d'affaires en faveur du libre marché est forte et conditionne l'intervention du gouvernement. Elle permet au secteur privé de s'assurer que la nouvelle compagnie ne contrarie pas l'activité des sociétés existantes. Ces interventions aboutissent à des limitations importantes. Toute immixtion de la compagnie dans le domaine de la distribution est considérée comme une superfétation, " une concurrence officielle à un commerce libre et privé ${ }^{8}$ ». Le gouvernement, quant à lui, anticipe la création d'une compagnie sans limitations, en mesure d'assurer l'approvisionnement de la France et l'indépendance de la nation. C'est ce qu'exige Louis Pineau, principal négociateur et directeur de l'Office national des combustibles liquides (ONCL). «Il faut que la société puisse faire tout ce que fait normalement une société de pétrole ${ }^{9}$. " Le gouvernement négocie pour qu'elle conserve toute sa liberté d'action, mais vu la situation du Trésor il a aussi besoin des capitaux privés. La plupart des efforts déployés par la Standard visent à empêcher la CFP de raffiner elle-même son pétrole. Elle avance que le consommateur français paiera son essence plus cher pour favoriser l'industrie de raffinage française. Ces négociations montrent l'influence déterminante qu'exercent les intérêts étrangers dans la formulation d'une politique nationale française. La création de la compagnie, en février 1924, ne résout pas complètement ces questions. Le gouvernement insiste surtout sur le caractère national de la société, les administrateurs seront français et leur nomination sera sujette à l'approbation du gouvernement ${ }^{10}$.

La création de la Compagnie française des pétroles est un jalon important de la mise en place d'une politique pétrolière nationale en France. Elle montre la complexité des rapports entre gouvernements et milieux d'affaires, entre national et international. Vers la fin des années 1920, les États-Unis contrôlent 60 \% de la production mondiale, ses systèmes d'exploitation, de développement et de distribution s'étendent à travers le globe. Dans le secteur de l'énergie, le retard pris par certaines puissances industrielles pousse les gouvernements à agir. La notion de sécurité nationale s'insère désormais dans les politiques économiques et industrielles, dont l'objectif est de palier aux carences de l'économie nationale. En France et ailleurs, la création de monopoles étatiques menace sérieusement la politique américaine en matière de commerce et d'investissement. Cette situation justifie l'intervention américaine dans les affaires nationales des pays consommateurs. La politique française risque de remettre en cause

\footnotetext{
${ }^{7}$ Archives de la banque Paribas, PTC/514, Compagnie nationale des pétroles, réunion tenue au ministère des Finances, 3 octobre 1923, p. 3.

${ }^{8}$ Ibid., Compte rendu de la réunion du comité de direction, 29 janvier 1924, p. 2.

${ }^{9}$ Ibid., PTC/515, Visite de M. Chevalier et de M. Martin à M. Hurll, 16 février 1924, p. 1.

${ }^{10}$ Archives de la compagnie Total, 89.14/-22, Maurice Mercier, l'œuvre et le rôle de la Compagnie française des pétroles, p. 5.
} 
la mise en place d'un ordre international libéral. Dans ce contexte, l'influence américaine plane autour de la création d'une compagnie nationale sur laquelle elle parvient à imposer d'importantes limitations. Or, l'évolution de la situation économique, les relations internationales et la croissance de la demande pétrolière remettent en question la politique initiée en 1924 par le gouvernement. La création d'une société nationale ne remet pas en question la mainmise qu'exercent les intérêts étrangers sur le secteur pétrolier. Le gouvernement français ne possède aucun moyen d'intervention à ce niveau. Or, l'acquisition d'un pétrole « français » nécessite qu'une part du marché lui soit attribuée.

Le besoin de contrôler le marché national est directement lié à la politique internationale mise en place par le gouvernement français. Seuls, les distributeurs français ne peuvent concurrencer les puissants trusts du pétrole. « Ce ne furent ni l'économie dirigée ni la modernisation, mais la fin des contrôles et le repli sur soi qui caractérisèrent la politique du régime après la guerre » (Kuisel, 1984 :122). C'est effectivement un retour à la liberté de commerce qui est mis en place en 1921, une décision qui pousse les compagnies étrangères à acheter les sociétés de distribution françaises pour prendre le contrôle du marché. La situation devient rapidement chaotique alors qu'une véritable guerre oppose les compagnies pour la conquête du marché intérieur. En janvier 1924, une première loi vise à mettre fin à l'anarchie. «L'importation en gros du pétrole ne pouvait être effectuée qu'en vertu d'une autorisation préalable délivrée à tout importateur remplissant les conditions exigées : double obligation de constituer des stocks de réserves et de fourniture aux services publics » (Murat, 1969: 30). C'est le régime de « liberté contrôlée », qui ne parvient pas «à mettre fin au contingentement privé imposé sur le marché intérieur par les maisons les plus puissantes » (Murat, 1969:30). Le 4 avril 1926, une nouvelle loi insiste sur la nécessité d'un monopole établi par l'État, son article 53 met l'accent sur les autorisations d'importations. La loi du 30 mars 1928 en est l'héritière et compte mettre l'article 53 en application. Elle renverse le régime de liberté conditionné mis en place plus tôt. L'État se dote ainsi d'une arme juridique pour réglementer le marché pétrolier français. C'est l'instauration du «monopole délégué »: grâce à un système d'autorisations spéciales le gouvernement contrôle l'importation, la distribution et le raffinage. Ces mesures complètent la volonté du gouvernement de se doter d'outils d'intervention dans l'économie. Elles permettront à la Compagnie française des pétroles de vendre et de raffiner ses produits sur le sol français.

\section{Conclusion}

La Première Guerre mondiale a fait émerger le caractère stratégique du pétrole. Cette prise de conscience conduit le gouvernement français à l'adoption de politiques économiques et industrielles dont l'impact est large. En France, les enjeux de ce secteur sont donc marqués du sceau du politique. À l'échelle nationale, la réglementation apparaît 
nécessaire pour assurer une répartition équitable des produits pétroliers. À l’international, les ententes diplomatiques permettent à l'Hexagone d'acquérir des gisements en Roumanie et au Moyen-Orient. Ces acquis se font aux dépens des intérêts allemands séquestrés pendant la guerre. La création d'une société nationale est le deuxième jalon de la politique pétrolière menée par le gouvernement après la guerre. Cette compagnie est de loin la plus importante société mixte créée durant la période. Toutes ces mesures témoignent de l'importance grandissante d'un certain nationalisme économique. La guerre érige de nouvelles frontières économiques à une échelle jamais connue auparavant. C'est l'installation d'une mentalité prédatrice sur la scène internationale. Il s'agit de remplacer les intérêts des puissances vaincues dans le monde et d'hériter de puissants atouts économiques. C'est précisément l'objectif du Quai d'Orsay dans le cadre des grandes tractations pétrolières. L’influence américaine est déterminante lors de l'adoption de nouvelles politiques. De manière générale, les États-Unis font preuve d'un manque d'empathie envers la mise en place de politiques nationalistes, en France et à l'étranger. Un fait remarquable pour une nation ayant employé des politiques protectionnistes afin de favoriser son propre développement économique tout au long du siècle précédent (Randall, 2005: 43).

Le contexte pétrolier de ces années ne va pas sans rappeler certaines analogies avec le temps présent, alors que les États-Unis cherchent de nouveau à remettre en question l'ordre économique mondial. À l'inverse de la situation actuelle, durant l'entre-deuxguerres, la compétitivité de l’industrie américaine lui assure la domination des marchés dans un contexte économique libéral. Par le biais de la politique de la Porte ouverte, Washington cherche donc à ouvrir les marchés mondiaux à son industrie. C'est précisément ce que tente d'éviter Londres, car son Empire lui garantit un accès privilégié à de nombreuses régions du globe. La France accuse un certain retard dans ce secteur ; malgré des millions dépensés en recherche, son empire ne lui fournit pas une goutte de pétrole durant l'entre-deux-guerres. C'est par le biais d'une diplomatie active et l'élaboration de politiques nationales qu'elle parvient à rattraper son retard. Ces interventions s'insèrent dans la volonté de moderniser l'économie française et d'assurer la compétitivité industrielle du pays

\section{Bibliographie}

Bouguen J.-M. (2013), Le pétrole en France, Paris, L’Harmattan.

Bull-Berg J. H. (1987), American International Oil Policy: Causal Factors and Effect, New York, St. Martin’s Press.

KuISEL R. (1967), Ernest Mercier French Technocrat, Berkeley, University of California Press.

KUISEL R. (1984), Le capitalisme et l'État en France. Modernisation et dirigisme au XX ${ }^{e}$ siècle, Paris, Gallimard. 
Marguerat P., JileK L. (1987), Banque et investissement industriel. Paribas, le pétrole roumain et la politique française, 1919-1939, Neuchâtel, Université de Neuchâtel, Genève, Librairie Droz.

MuRAT D. (1969), L'Intervention de l'État dans le secteur pétrolier en France, Paris, Éditions Technip.

Pelletier F. (2016), « Paribas en Roumanie, influence bancaire et impératifs politiques », Guerres mondiales et conflits contemporains, vol. 263, $\mathrm{n}^{\circ} 3$, p. 105-122.

Pelletier F. (2017) « La France et l’industrie pétrolière roumaine », Histoire, économie et société, vol. 36, $\mathrm{n}^{\circ}$ 3, p. 87-104.

NAYBERG R. (1997), « Qu'est-ce qu'un produit stratégique ? L'exemple du pétrole », Défense nationale, vol. 584, p. 77-86.

Randall S. (2005), United States Foreign Oil Policy Since World War I, Montréal, McGillQueen's University Press.

SANDU T. (1999), La Grande Roumanie alliée de la France. Une péripétie des Années Folles? (1919-1933), Paris, L'Harmattan (« Aujourd'hui l'Europe »).

VAISSET T. (2012), « Du charbon au mazout. La révolution de la chauffe dans la Marine nationale », in A. BELTRAN, Le pétrole et la guerre, Bruxelles, Bern, Berlin, Frankfurt, New York, Oxford, Wien, Peter Lan 